\title{
Novel bioinformatic classification system for genetic signatures identification in diffuse large B-cell lymphoma
}

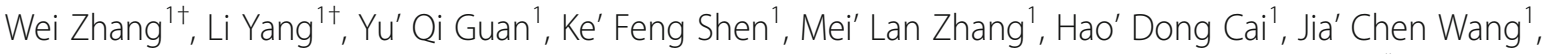 \\ Ying Wang ${ }^{1}$, Liang Huang ${ }^{1}$, Yang Cao ${ }^{1}$, Na Wang ${ }^{1}$, Xiao' Hong Tan², Ken He Young ${ }^{3}$, Min Xiao ${ }^{1 *}$ and \\ Jian' Feng Zhou'
}

\begin{abstract}
Background: Diffuse large B-cell lymphoma (DLBCL) is a spectrum of disease comprising more than $30 \%$ of nonHodgkin lymphomas. Although studies have identified several molecular subgroups, the heterogeneous genetic background of DLBCL remains ambiguous. In this study we aimed to develop a novel approach and to provide a distinctive classification system to unravel its molecular features.

Method: A cohort of 342 patient samples diagnosed with DLBCL in our hospital were retrospectively enrolled in this study. A total of 46 genes were included in next-generation sequencing panel. Non-mutually exclusive genetic signatures for the factorization of complex genomic patterns were generated by random forest algorithm.

Results: A total of four non-mutually exclusive signatures were generated, including those with MYC-translocation (MYC-trans) ( $n=62)$, with BCL2-translocation (BCL2-trans) $(n=69)$, with BCL6-translocation (BCL6-trans) $(n=108)$, and those with MYD88 and/or CD79B mutations (MC) signatures $(n=115)$. Comparison analysis between our model and traditional mutually exclusive Schmitz's model demonstrated consistent classification pattern. And prognostic heterogeneity existed within EZB subgroup of de novo DLBCL patients. As for prognostic impact, MYC-trans signature was an independent unfavorable prognostic factor. Furthermore, tumors carrying three different signature markers exhibited significantly inferior prognoses compared with their counterparts with no genetic signature.
\end{abstract}

Conclusion: Compared with traditional mutually exclusive molecular sub-classification, non-mutually exclusive genetic fingerprint model generated from our study provided novel insight into not only the complex genetic features, but also the prognostic heterogeneity of DLBCL patients.

Keywords: DLBCL, Sequencing, Random forest, Classification, Signature

\section{Background}

Diffuse large B-cell lymphoma (DLBCL) is the most prevalent lymphoid malignancy in adult patients, comprising $30-40 \%$ of non-Hodgkin lymphomas [1].

\footnotetext{
* Correspondence: xiaomin@tjh.tjmu.edu.cn

+Wei Zhang and Li Yang contributed equally to this work.

'Department of Hematology, Tongji Hospital, Tongji Medical College,

Huazhong University of Science and Technology, Wuhan 430030, Hubei Province, P.R. China

Full list of author information is available at the end of the article
}

Although durable remissions can be achieved in a substantial proportion of patients after chemoimmunotherapy with rituximab, cyclophosphamide, doxorubicin, vincristine, and prednisone (R-CHOP), over $30 \%$ of cases develop refractory or relapsed disease [2]. Previous studies have revealed that DLBCL is a genetically heterogeneous disorder with considerable gene mutations, copy number $(\mathrm{CN})$ alterations, and structural variants [3-5].

(c) The Author(s). 2020 Open Access This article is licensed under a Creative Commons Attribution 4.0 International License, which permits use, sharing, adaptation, distribution and reproduction in any medium or format, as long as you give appropriate credit to the original author(s) and the source, provide a link to the Creative Commons licence, and indicate if changes were made. The images or other third party material in this article are included in the article's Creative Commons licence, unless indicated otherwise in a credit line to the material. If material is not included in the article's Creative Commons licence and your intended use is not permitted by statutory regulation or exceeds the permitted use, you will need to obtain permission directly from the copyright holder. To view a copy of this licence, visit http://creativecommons.org/licenses/by/4.0/. The Creative Commons Public Domain Dedication waiver (http://creativecommons.org/publicdomain/zero/1.0/) applies to the data made available in this article, unless otherwise stated in a credit line to the data. 
Understanding the molecular basis of this heterogeneity may facilitate individualized management strategies.

Researchers have focused on developing a robust algorithm to discover distinct subsets and subclassify DLBCLs. In 2000, based on gene expression profiling results, Alizadeh et al. identified two molecularly distinct forms of DLBCL, germinal center B-cell-like (GCB) and activated $B$-cell-like $(A B C)$, representing different stages of B-cell differentiation [6]. Nevertheless, according to the cell-oforigin (COO) classification, approximately $10 \% \sim 20 \%$ of DLBCLs remain unclassified, and the molecular pathogenesis of DLBCL remains obscure. The rapid development of next-generation sequencing (NGS) technology has revealed accumulated recurrent genetic alterations, which have improved our understanding of the genetic landscape of DLBCL. However, these genomic studies had largely focused on single types of alterations. In 2018, Schmitz et al. studied tumor specimens from 574 patients with DLBCL [4]. By developing a subclassified algorithm based on coding region mutations, $\mathrm{CN}$ variations, and structure variations (SVs), they further identified four distinct genetic subtypes of DLBCL, which included BN2 (based on BCL6 fusions and NOTCH2 mutations), N1 (based on NOTCH1 mutations), $\mathrm{MCD}$ (based on the co-occurrence of $M Y D 88^{\mathrm{L} 265 \mathrm{P}}$ and $C D 79 B$ mutations), and EZB (based on $E Z H 2$ mutations and $B C L 2$ translocations). Chapuy et al. also carried out a comprehensive genetic analysis of 304 primary DLBCLs and identified five subgroups of DLBCLs with prominent genetic features $(\mathrm{C} 1-\mathrm{C} 5)$, [5]. These studies provided us with a novel roadmap for an actionable DLBCL classification for precision-medicine-based strategies in DLBCL.

Although several specific subgroups, such as primary DLBCL of the central nervous system (PCNSL), primary mediastinal (thymic) large B-cell lymphoma (PMBL), primary cutaneous DLBCL, leg type (PCDLBCL-LT), high-grade B-cell lymphoma, not otherwise specified (HGBL, NOS), and HGBL with $M Y C$ and BCL2 and/or BCL6 translocations (HGBL-DH/TH), are all welldefined entities, they share clinicopathologic features and genetic alterations with DLBCL, NOS according to previous studies [7-10]. In fact, they belong to a disease spectrum rather than discrete entities. In 2018, Scott et al. defined a clinically and biologically distinct subgroup of tumors within GCB DLBCL characterized by a gene expression signature of HGBL-DH/TH-BCL2 [11]. Simultaneously, Westhead et al. also defined a molecular high-grade (MHG) group by applying a gene expression-based classifier [12]. Chapuy et al. also mentioned in their study that molecular heterogeneity existed within the C3 subgroup [5]. Their studies suggest that beyond current definitions of double- and triple-hit DLBCLs, more specified molecular subgroups of DLBCL requires further exploration.
In this study, instead of mutually exclusive subclassification, we determined several non-mutually exclusive genetic signatures for the factorization of complex genomic patterns in a continuous spectrum of Bcell lymphomas based on a random forest algorithm. Using this model, we also presented a single-center primary site-relevant mutational pattern based on the Chinese population. This study aims to develop a novel approach to understand the molecular features, provide a distinctive insight nosologically, and orient targeted therapeutic strategies and prognostic evaluation of the spectrum of large B-cell lymphomas.

\section{Methods}

\section{Patients and samples}

This study was approved by the institutional review board of Tongji Hospital. From June 2008 to September 2018, a cohort of 342 patient samples diagnosed with DLBCL in our hospital were retrospectively enrolled in this study. All cases were reviewed by at least three experienced hematopathologists. Most samples were pretreatment biopsies from de novo cases $(n=298)$, including DLBCL, NOS $(n=239)$, PMBL $(n=13)$, PCNS L $(n=7)$, PCDLBCL-LT $(n=6)$, HGBL, NOS $(n=7)$, and HGBL-DH/TH $(n=26)$. The remainder consisted of relapsed DLBCL, NOS $(n=25)$ after R-CHOP or CHOP-like chemotherapy and transformed follicular lymphoma (tFL) samples $(n=19)$. All samples were obtained from formalin-fixed paraffin embedded (FFPE) tissue. Tumor content was estimated to be at least 30\% in all subjects. Genomic DNA was extracted with the GeneRead $^{\text {tw }}$ DNA FFPE Kit (Qiagen) according to the manufacturer's instructions.

DLBCL samples were classified into GCB and nonGCB subtypes by the IHC-based Hans algorithm [13, 14]. Translocations in $M Y C, B C L 2$, and BCL6 and $C N$ aberrations in TP53 were examined by performing fluorescent in situ hybridization (FISH). Cases were excluded if $\mathrm{COO}$ subtype or FISH findings were not available. Clinical data, including age group of onset, International Prognostic Index (IPI), primary site of lymphomagenesis, chemotherapy regimen, initial response to therapy, overall survival (OS), and progression-free survival (PFS), were collected (Table 1) (Supplementary Table S1). We restrictively included individuals in which biopsy tissue was evaluated as the primary site of lymphomagenesis, according to integrated judgment based on PET-CT scanning, pathological findings, and clinical manifestation [15]. Criteria of response assessment and definition of OS and PFS were followed by the Lugano Classification [16]. Only patients receiving $\mathrm{R}-\mathrm{CHOP}$ or $\mathrm{R}-\mathrm{CHOP}-$ like regimens were included in the prognostic analyses. Multivariable Cox proportional hazard regression 
Table 1 Baseline features of 342 cases included in this study

\begin{tabular}{|c|c|c|c|c|c|c|c|c|}
\hline \multirow[t]{2}{*}{ Parameters } & \multicolumn{6}{|c|}{ De novo cases $(n=298)$} & \multirow{2}{*}{$\begin{array}{l}\text { Relapsed cases } \\
\text { DLBCL, NOS } \\
(n=25)\end{array}$} & \multirow{2}{*}{$\begin{array}{l}\text { Transformed } \\
\text { cases } \\
\text { tFL }(n=19)\end{array}$} \\
\hline & $\begin{array}{l}\mathrm{DLBCL}, \mathrm{NOS} \\
(n=239)\end{array}$ & $\begin{array}{l}\mathrm{PMBCL} \\
(n=13)\end{array}$ & $\begin{array}{l}\text { PCNSL } \\
(n=7)\end{array}$ & $\begin{array}{l}\text { PCDLBCL-LT } \\
(n=6)\end{array}$ & $\begin{array}{l}\text { HGBL, NOS } \\
(n=7)\end{array}$ & $\begin{array}{l}\mathrm{HGBL}-\mathrm{DH} / \mathrm{TH} \\
(n=26)\end{array}$ & & \\
\hline \multicolumn{9}{|l|}{ Gender } \\
\hline Male & 131 & 6 & 6 & 2 & 5 & 9 & 16 & 15 \\
\hline Female & 108 & 7 & 1 & 4 & 2 & 17 & 9 & 4 \\
\hline \multicolumn{9}{|l|}{ Age of onset } \\
\hline Median (years) & 52 & 32 & 59 & 68 & 60 & 50 & 56 & 48 \\
\hline \multicolumn{9}{|l|}{ COO subtype } \\
\hline GCB & 95 & 6 & 0 & 2 & 4 & 23 & 12 & 14 \\
\hline Non-GCB & 144 & 7 & 7 & 4 & 3 & 3 & 13 & 5 \\
\hline \multicolumn{9}{|c|}{ Type of biopsy tissue } \\
\hline Nodal & 122 & 0 & 0 & 0 & 5 & 19 & 14 & 15 \\
\hline Extranodal & 117 & 13 & 7 & 6 & 2 & 7 & 11 & 4 \\
\hline \multicolumn{9}{|l|}{ IPI at first diagnosis } \\
\hline $0 \sim 1$ & 56 & 3 & 1 & 0 & 2 & 5 & 3 & N/A \\
\hline $2 \sim 3$ & 134 & 8 & 5 & 4 & 2 & 15 & 17 & N/A \\
\hline $4 \sim 5$ & 49 & 2 & 1 & 2 & 3 & 6 & 5 & N/A \\
\hline \multicolumn{9}{|c|}{ First chemotherapy regimen } \\
\hline $\begin{array}{l}\text { R-CHOP or R- } \\
\text { CHOP-like }\end{array}$ & 228 & 13 & 0 & 6 & 7 & 26 & 25 & 9 \\
\hline Other or N/A & 11 & 0 & 7 & 0 & 0 & 0 & 0 & 10 \\
\hline
\end{tabular}

Abbreviations: DLBCL, NOS Diffuse Large B cell Lymphoma, Not Otherwise Specified, PMBCL Primary Mediastinal B-cell Lymphoma, PCNSL Primary Central Nervous System Lymphoma, PCDLBCL-LT Primary Cutaneous Diffuse Large B Cell Lymphoma, Leg Type, HGBL, NOS High Grade B-Cell Lymphoma, Not Otherwise Specified, HGBL-DH/TH High-Grade B-cell Lymphomas harboring rearrangements of MYC and BCL2 and/or BCL6, tFL Transformed Follicular Lymphoma, GCB Germinal Center B-cell-like, IPI International Prognostic Index, R-CHOP Rituximab plus Cyclophosphamide, Doxorubicin, Vincristine, and Prednisone;

models were used to evaluate proposed prognostic factors.

\section{Targeted high-throughput sequencing}

A total of 46 genes were selected in this study (Supplementary Table S2). Most genes were frequently altered in DLBCL according to data from several previously published large-scale DLBCL cohort studies [3-5]. Additionally, several infrequently mutated genes were also included because they are specifically related to several DLBCL subtypes. In detail, ID3, TCF3, DDX3X are related with double-hit lymphoma [17]/Burkitt lymphoma [18]; XPO1 is related with primary mediastinal large Bcell lymphoma [8]; RRAGC, POU2AF1 are related with Follicular lymphoma [19]; KLF2 is related with marginal zone lymphoma [20]. Besides, in our panel we also selected genes functioning in signaling pathways which are crucial for DLBCL pathogenesis (e.g. FBXW7 for NOTCH pathway; NRAS, KRAS, MAP $2 \mathrm{~K} 1$ for MAPK pathway). Using genome build hg19/GRCh37 as a reference, a sequencing panel covering the coding sequences (CDS) within 5 intronic base pairs around exons in 46 genes was designed online (Designstudio Sequencing,
Illumina, San Diego, USA). Sequencing libraries were prepared with AmpliSeq ${ }^{\mathrm{Tm}}$ Library PLUS for Illumina, using $20 \mathrm{ng}$ of input genomic DNA per sample. Library sequencing was performed to $2000 \times$ coverage on a NextSeq $^{\mathrm{Ts}} 550$ system using an Illumina NextSeq ${ }^{\mathrm{Tm}} 500 / 550$ High Output v2 Kit (Illumina, San Diego, USA). The alignment and variant calling were performed using a DNA Amplicon workflow with default parameters on BaseSpace Sequence Hub (Illumina). Generated variants were further annotated using Annovar [21].

Variant filtering was performed by the following cascade of steps: 1) select exon nonsynonymous or splice donor/acceptor site variants; 2) exclude variants with population frequency $>0.0001$ in the gnomAD database unless variant is included as a somatic variant of lymphoid neoplasm in the COSMIC database; 3) exclude variants present in an in-house curated blacklist. The formation of our variant screening blacklist was based on the idea described previously by Schmitz et al. [4] As these false positive variants were presumed to be artifacts generated either by the high throughput sequencing platform itself or due to errors in alignment or annotation of the sequencing reads by the analytical 
pipeline. Typically, these variants were abnormally prevalent, identified exclusively in specific sequencing platform, and are not recurrent variants included in the major public cancer somatic mutation database (COSMIC database, https://cancer.sanger.ac.uk/cosmic). Therefore, as such variants were unique in our center and there were no universal criteria for identification, the blacklist was built for future rapid and accurate variants' screening. Detailed variants information included in the blacklist were listed in Supplementary Table S7; 4) exclude variants found in regions with poor coverage; and 5) exclude variants with quality less than 30 or read depth less than 20. For activation-induced cytidine deaminase (AID) somatic hypermutation (SHM) analysis, we additionally selected synonymous variants and variants in intron/UTR regions, and each variant also needed to fulfill the aforementioned criteria from step 2 to step 5 [22].

Sanger sequencing in matched normal DNA was performed if it was available for each missense mutation that passed all preceding filters and met the following conditions: 1) variant allele frequency (VAF) more than 0.40 and less than $0.60 ; 2$ ) variant was not included in the COSMIC database; 3) variant was included in the gnomAD database. Confirmed germline mutations were excluded in further analyses.

\section{Fluorescent in-situ hybridization analysis}

Interphase fluorescence in situ hybridization (FISH) studies were performed using commercially available probes (Abbott Molecular, Downers, Grove, IL, USA). The LSI IGH/IGHV (14q32), and LSI MYC (8q24) Dual Color, Break Apart Rearrangement Probes were used to detect the rearrangement of BCL2, BCL6 and c-Myc respectively. A 17p13.1 (P53) probe (Vysis, Downers, Grove, IL) was used to detect $17 \mathrm{p}$ deletion. Sample preparations and hybridizations were conducted following the manufacturer's recommendations and 200 cells were analyzed for each probe.

\section{Bioinformatic algorithm}

Artificial Intelligence (AI) is the intelligence manifested by a human-made machine. It usually refers to the ability of a computer to simulate human thought processes in order to mimic human abilities or behaviors. AI not only deals with problems under pre-set rules, but also develops capabilities to generate judgements under new situations through feature identification. The performance of these feature-driven algorithms can improve as they are exposed to more data over time, which is similar to the human learning process. Therefore, such algorithms are named machine learning. Among existing learning methods, random forests are an ensemble learning method for classification or regression that operate by constructing a multitude of decision trees at training time and outputting the classification or regression of the individual trees. In this study, our model was trained using the $\mathrm{R}$ package 'randomForest'. The number of trees was set to 100; all other hyperparameters were set to their default values. Detailed information on the algorithm was described in Supplementary Appendix.

\section{Statistical analysis}

All statistical analyses were evaluated by R v3.5.1. Differences were analyzed using Fisher's exact test for categorical variables. The significance of the co-occurrence or mutual exclusivity was calculated using a Fisher exact test, for numerous tests, $p$ values were FDR - adjusted using Bonferroni method. The Kaplan-Meier method and log-rank test were used for survival analysis. Unless otherwise specified, a two-sided $P$-value $<0.05$ was considered statistically significant for all analyses.

\section{Results \\ Mutational profile through next-generation sequencing analysis}

A total of 1240 candidate variants were identified in 46 genes (Supplementary Table S3). Of 342 cases, 330 (96.5\%) harbored at least one mutation. Firstly, as for de novo DLBCL, NOS patients, the most frequently mutated genes were TP53 (28.9\%), PIM1 (27.2\%), KMT2D (25.9\%), MYD88 (23.0\%), and CD79B (22.6\%) (Supplementary Fig. S1), and variant frequency of MYD88 was significantly more frequent compared with relapse DLBCL cases $(P=0.014)$. As for relapse DLBCL cases, $K M T 2 D$ and KRAS mutations were significantly more frequent compared with those in de novo cases $(P=$ $0.031 ; P=0.012$ ). Secondly, as for gene functional groups, mutations in genes associated with chromatin remodeling, including $K M T 2 D$ and $C R E B B P$, and genes associated with apoptosis resistance, including $B C L 2$, were significantly enriched in cases of transformed follicular lymphoma (tFL) in comparison with de novo cases $\left(P=2.20 \times 10^{-4} ; P=3.51 \times 10^{-4} ; P=1.93 \times 10^{-5}\right)$. While immune evasion-related genes, including $B 2 M$, $C D 58$, and $C D 70$, were frequently detected in de novo cases $(16.3,12.1,9.6 \%)$ but not in tFL in our cohort $(P=$ $0.039 ; P=0.095 ; P=0.158)$. Thirdly, through comparison of mutation gene pattern differences among GCB and non-GCB DLBCL subgroups in all de novo, relapsed, and transformed cases, we determined that the $B C L 2$ translocation; the $M Y C$ translocation; and the CREBBP, TNFRSR14, BCL2, EZH2, SGK1, and ID3 mutations were significantly more frequent in GCB DLBCL $(P<0.001)$, whereas $C D 79 B$ mutations, the $M Y D 88^{\mathrm{L} 265 \mathrm{P}}$ mutation, and the $B C L 6$ translocation were more common in non-GCB DLBCL $(P<0.001)$ (Fig. 1a). 


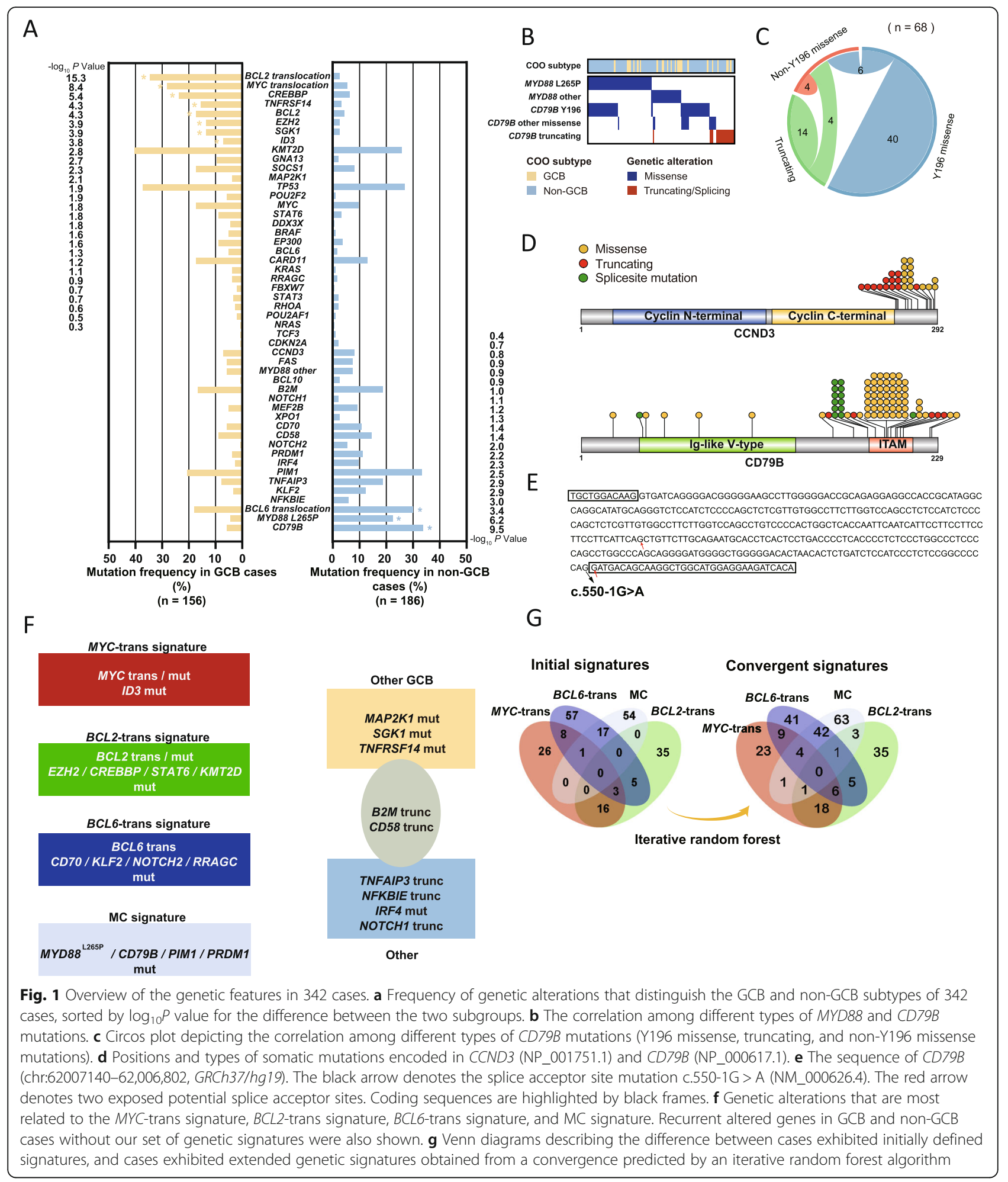

In addition, in this study we specifically focused on the analysis of $C D 79 B$ mutation pattern. In general, the variant frequency of $C D 79 B$ was relative higher compared with recent related studies [3-5, 23, 24]. In detail, for the typical hotspot variant $C D 79 B^{\mathrm{Y} 196}$, we found the top co-occurrent mutation with $C D 79 B^{\mathrm{Y} 196}$ was $M Y D 88^{\mathrm{L} 265 \mathrm{P}}$ $(n=37)$ (adjusted $P$ value $1.09 \times 10^{-9}$ ) in our de novo DLBCL, NOS cases, which was mainly identified in nonGCB subtype (4.5\% of GCB cases vs. $22.6 \%$ of non-GCB cases, $P=5.87 \times 10^{-7}$ ) (Fig. 1b). Meanwhile, we found 
that $C D 79 B$ non-Y196 codon mutations accounted for $41 \%(33 / 80)$ of all $C D 79 B$ mutations. Moreover, through validation Sanger sequencing of tumor and paired normal tissue DNA, we determined several novel hotspot intron splice site mutations, including c.550-1G $>\mathrm{A}$, c.550-1G > C, c.550-3_552del, c.549+1G > A, c. $549+$ $1 \mathrm{G}>\mathrm{C}$, and c.540_549+1del (Fig. 1d). Focusing on the molecular impact of c.550-1G > A mutation, we subsequently performed RNA sequencing and revealed that this mutation resulted in exposing two novel potential splice acceptor sites, thereby synthesizing two truncating proteins (Fig. 1e). Furthermore, we also found that $C D 79 B$ truncating mutations were mutually exclusive with $C D 79 B^{\mathrm{Y} 196}$ (adjusted $P$ value 0.011 ). Similar tendency was witnessed for $\mathrm{CD} 79 \mathrm{~B}$ truncating mutations with $M Y D 88^{\mathrm{L} 265 \mathrm{P}}$, while without statistical significance (adjusted $P$ value 0.14) (Fig. 1b, Fig. 1c).

\section{Identification of genetic signatures via iterative random forest (RF) algorithm}

In this study, based on targeted sequencing results and FISH findings, we attempted to identify several nonmutually exclusive representative genetic signatures instead of categorizing subjects into several mutually exclusive distinct subgroups. Therefore, we decided to seed our analysis from 5 genetic alterations which participated in the most important cellular signaling pathways in DLBCL pathogenesis, i.e. cellular proliferation (MYC translocation), apoptosis resistance (BCL2 translocation), immune cell differentiation abruption (BCL6 translocation) and activation of inflammation pathway (CD79B ${ }^{\mathrm{Y} 196}$ and MYD88 ${ }^{\mathrm{L} 265 \mathrm{P}}$ ). Moreover, all five genetic alterations were specifically enriched in either GCB or nonGCB subtype DLBCL patients ( $>20 \%$ positive in GCB or non-GCB DLBCL patients). In addition, these alterations exhibited most distinctive frequencies between GCB and non-GCB DLBCL subtypes by Fisher's test (Fig. 1a). Thus, using the five main features above, we initially defined four non-mutually exclusive genetic signatures: 1 ) the $M Y C$-trans signature, with $M Y C$ translocation $(n=$ $54)$; 2) the $B C L 2$-trans signature, with $B C L 2$ translocation $(n=59)$; 3$)$ the BCL6-trans signature, with BCL6 translocation $(n=91)$; and 4$)$ the MC signature, with $M Y D 88^{\mathrm{L} 265 \mathrm{P}}$ and/or $C D 79 B^{\mathrm{Y} 196}$ mutations $(n=72)$ (Fig. 1f).

Among the above-mentioned four signatures, $\mathrm{MC}$ signature combined CD79B ${ }^{\mathrm{Y} 196}$ and MYD88 ${ }^{\mathrm{L} 265 \mathrm{P}}$ variants as they not only presented as hotspot mutations in DLBCL patients, but also exhibited statistically significant tendency for co-occurrence (adjusted $P$ value $\left.1.09 \times 10^{-9}\right)$. In addition, previous researches also revealed that both variants resulted in constitutive activation of NF- $k B$ signaling pathway [5]. Inspired by the study conducted by R. Schmitz et al., we aimed to evolve and maximize each genetic signature with our set of genetic features while appropriately maintaining the pattern suggested by the initial genetic signature. To alleviate such semisupervised problems, we developed an iterative random forest (RF) algorithm (Supplementary Appendix). The label of each genetic signature among cases gradually propagated and obtained convergence (Supplementary Table S4; Fig. 1g). Additionally, 8 (14.8\%), 10 (16.9\%), 17 (18.7\%), and 43 (59.7\%) cases were predicted to exhibit the $M Y C$-trans, BCL2-trans, $B C L 6$-trans, and $M C$ signatures, respectively, suggesting that the initial definition of the MC signature might be conservative. As a result, 252 out of 342 cases (73.7\%) were finally confirmed to be associated with at least one genetic signature.

Next, we investigated other genetic mutations statistically associated with one of these genetic signatures. As illustrated in Fig. 2, genetic mutations of each case were combined and clustered within different genetic signatures, and were shown in factorized mutational heatmap. Firstly, $M Y C$ and ID3 mutations were associated with the $M Y C$-trans signature $(P<0.001)$, and $40 \%(8 / 20)$ of cases with isolated $M Y C$-trans signatures harbored mutations in the ID3-TCF3-CCND3 pathway. We also recognized that all $M Y C$ hypermutations were identified in cases with $M Y C$-trans signatures (20/20, 100\%), while $M Y C$ non-hypermutations were common in cases with either MYC-trans signatures $(10 / 25,40 \%)$ or BCL6-trans signatures $(15 / 25,60 \%)$. Secondly, $B C L 2, E Z H 2$, CREBBP, STAT6, and KMT2D mutations were significantly related to the $B C L 2$-trans signature $(P<0.001)$. Although the $B C L 2$ mutation was associated with the $B C L 2$-trans signature, cases harboring the $B C L 2$ hypermutation usually implied that they had a combined $M Y C$-trans and BCL2-trans signature (6/6, 100\%). For chromatin modification-associated genes such as $K M T 2 D$ and $C R E B B P$, cases harboring co-occurring mutations in KMT2D and CREBBP generally indicated a $B C L 2$-trans signature $(21 / 24,87.5 \%)$. Thirdly, for the BCL6-trans signature, the CD70, KLF2, NOTCH2, and RRAGC mutations were specifically identified $(P<$ 0.001). Although the CCND3 mutation was more specifically associated with the $M Y C$-trans signature $(P=$ 0.001 ), it was also frequent in cases with the BCL6-trans signature $(16 / 108,14.8 \%)$. A vast majority of $K L F 2$ zinc finger mutations $(15 / 22,68.2 \%)$ were identified in cases with BCL6 translocation (or BCL6-tran signature, 21/22, $95.5 \%$ ), which had not been previously reported. Finally, for the MC signature, in addition to the $C D 79 B^{\mathrm{Y} 196}$ and $M Y D 88^{\mathrm{L} 265 \mathrm{P}}$ mutations, other types of mutations, such as PIM1 and PRDM1, were also significantly related to the MC signature $(P<0.001) . X P O 1$ E571K, a hotspot mutation in chronic lymphocytic leukemia (CLL) and PMBL, was also frequently identified in cases with MC 


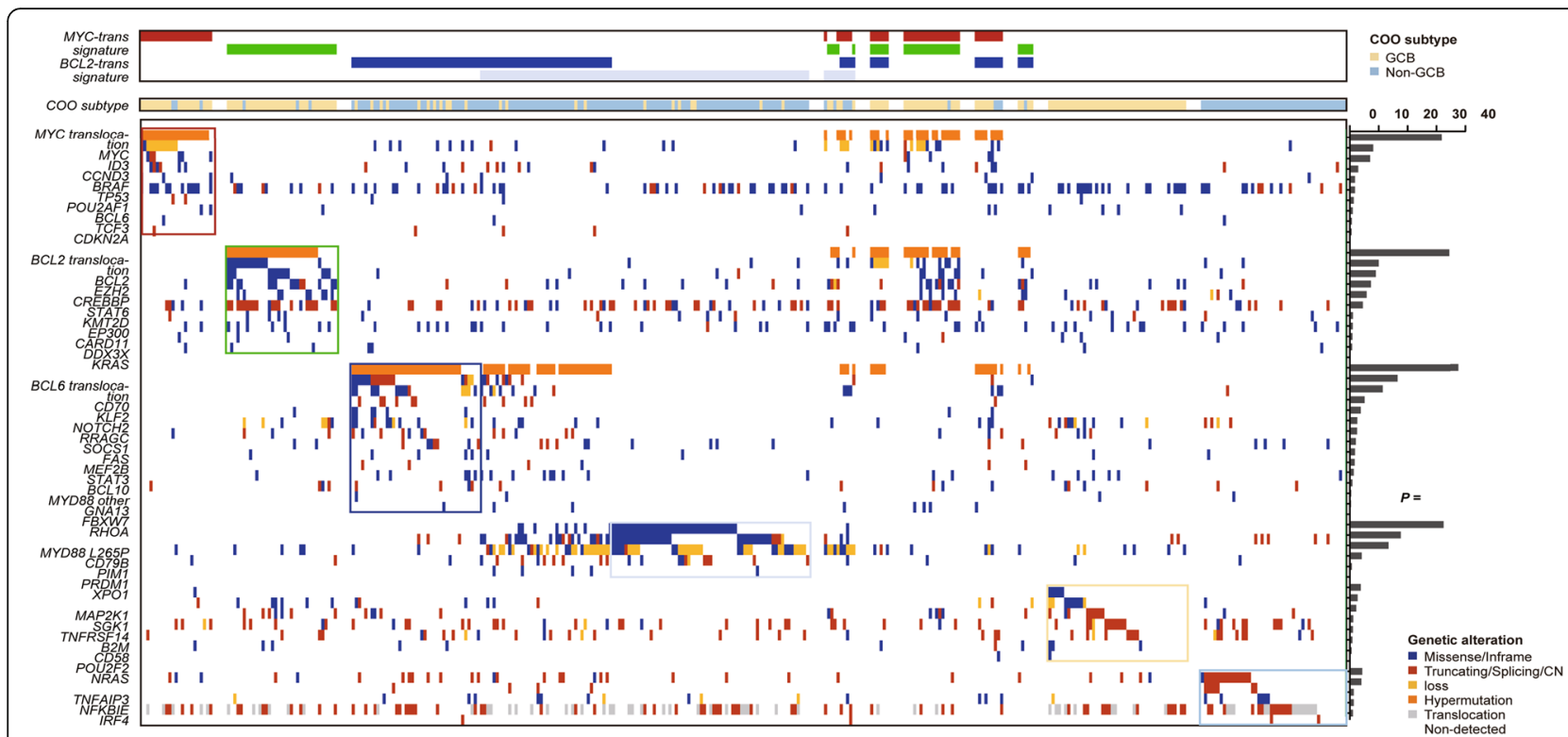

Fig. 2 Schematic of the association between genetic alterations and genetic signatures. All 342 cases were clustered and arranged according to the absent/present status of four genetic signatures. We determined the prevalence of each genetic alteration in the following six subsets: 1) cases presented isolated MYC-trans signatures, 2) cases presented isolated BCL2-trans signatures, 3) cases presented isolated BCL6-trans signatures, 4) cases presented isolated MC signatures, 5) GCB cases without any genetic signatures, and 6) non-GCB cases without any genetic signatures. Genetic alterations were thus clustered into six corresponding classes depending on their maximum prevalence among the six subsets. Genetic alterations in the same cluster were ranked by the significance between cases with isolated corresponding genetic signatures and cases without corresponding genetic signatures (or "other GCB"/"non-GCB" vs. the remaining), with $\log _{10} P$ value depicted to the right of the factorized heatmap. Color code of genetic alteration types: missense mutation or in-frame deletion/insertion (blue), truncating mutation, splice donor/ acceptor site mutation, or copy number loss in TP53 (red), SHM (yellow), translocation (orange), and nondetected (gray). COO classification was also indicated above the factorized heatmap

signatures and was usually accompanied by the BCL6trans signature $[25,26]$.

\section{Model comparison with classical DLBCL subtype classifier and its prognostic significance}

In order to validate our genetic classification algorithm, we compared our model with the classical DLBCL genetic classifier built from Schimtz et al. [4] for 239 de novo DLBCL NOS cases in our study cohort. As illustrated from Fig. 3a, $65 \%$ of all cases $(n=155)$ were successfully classified into four genetic subtypes (MCD $n=$ 66 , BN2 $n=55$, EZB $n=30$, N1 $n=4)$. In comparison, $75 \%$ of all cases $(n=175)$ could be classified in at least one signature subtype. $\mathrm{COO}$ classification also demonstrated similar type distribution (GCB and non-GCB) between two models (Fig. 3b). As for each genetic subtype of Schimtz et al. (Fig. 3c), the majority of cases within MCD subtype could be grouped in MC-trans signature (63 of 66, 95.4\%), and the consistent result was seen in BCL6-trans signature within BN2 subtype (54 of 55, 98.2\%) and BCL2-trans signature within EZB subtype (29 of 30, 96.7\%). However, in addition to the consistency between two models mentioned above, we did find that a portion of the DLBCL cases within each subtype of Schimtz's model carried 2 or more signatures.
In detail, within MCD and BN2 subtypes, 15 out of 66 (22.7\%) and 19 out of 55 (34.5\%) patients carried both MC-trans and BCL6-trans signatures, respectively. While in EZB subtypes, 6 out of 30 (20.0\%) patients carried both BCL2-trans and BCL6-trans signatures.

To evaluate the prognostic value of our genetic subtype model, we selected all de novo patients with large B-cell lymphoma who received $\mathrm{R}-\mathrm{CHOP}$ or $\mathrm{R}-\mathrm{CHOP}$ like chemotherapy $(n=280$, maximum follow-up 60 months, median follow-up 26 months). We next constructed a multivariate Cox proportional hazard regression model considering both genetic signatures and IPI scores as variables. The $M Y C$-trans signature was the most unfavorable genetic signature, and the $M Y C$-trans signature had a hazard ratio (HR) of 2.00 compared with the absented $M Y C$-trans signature (OS: $P=0.006$ ) (Supplementary Table S5). Those who presented a $B C L 2$ trans signature had a relatively favorable 5 -year PFS, with a borderline significance $(P=0.087)$. According to the non-mutually exclusive nature of our set of four genetic signatures and several latest research achievements $[3,5,11,12,27]$, we aimed to explore the differences in prognostic impact for de novo DLBCL cases with various genetic signature numbers. Firstly, in order to exclude the potential influences of confounding factors, 




especially IPI score, we examined the statistical differences of IPI score group distribution (low 0-1, intermediate 2-3, high 4-5) between groups of patients with varying number of genetic signatures (0-sig, 1-sig, 2-sig, 3 -sig). As a result, no statistical differences of IPI level distribution were identified between 0-.

Sig, 1-sig, 2-sig and 3-sig patient groups ( $p>0.05$, Chisquare and Fisher Exact test with Bonferroni adjustment). As reflected by the 5-year OS and PFS time (Fig. 4a-b), we found that individuals carrying three signatures had much worse prognosis than individuals without any genetic signature (OS: $P=0.0084$; PFS: $P=$ 0.3274 ), while patients with only one genetic signature exhibited no significant difference in prognosis compared with those without any signature (Fig. 4c-d). In addition, further subgroup survival analysis indicated that within EZB subtypes of Schmitz model, patients carrying BCL2-trans plus BCL6-trans or MC-trans signatures exhibited significantly inferior prognosis, compared with patients carrying BCL2-trans signature only (OS: $P=0.002$; PFS: $P=0.039$ ) (Fig. 4e-f). However, no prognostic differences were identified in patients carrying different number of signatures within $\mathrm{MCD}, \mathrm{BN} 2$ or $\mathrm{N} 1$ subgroups. The above findings provided evidence that these non-mutually exclusive genetic signatures exhibited cumulative prognostic influences, and patient heterogeneity still existed in traditional mutually exclusive classification model for DLBCL patients in our cohort, which requires further confirmation in larger multi-center cohort studies.

\section{Discussion}

In this study we retrospectively analyzed NGS sequencing results of DLBCL cohort. Among all panel genes sequenced, we focused on the variant pattern of $C D 79 B$. In general, variant frequency of $C D 79 B$ was relative higher comparing with reports from other centers, which could be possibly explained with ethnical difference and 


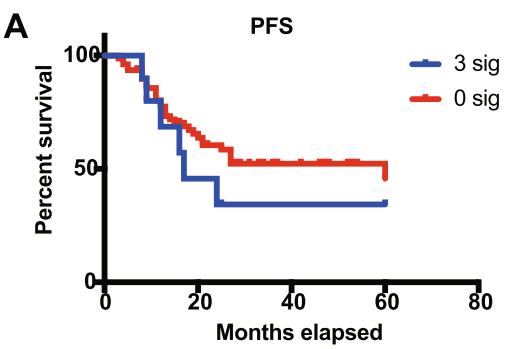

C



E

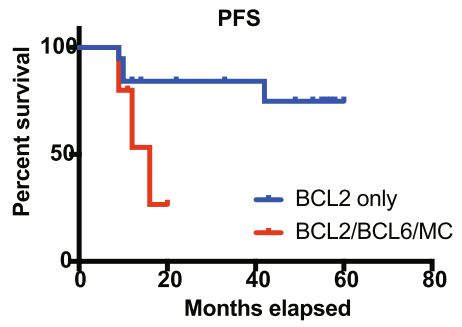



D

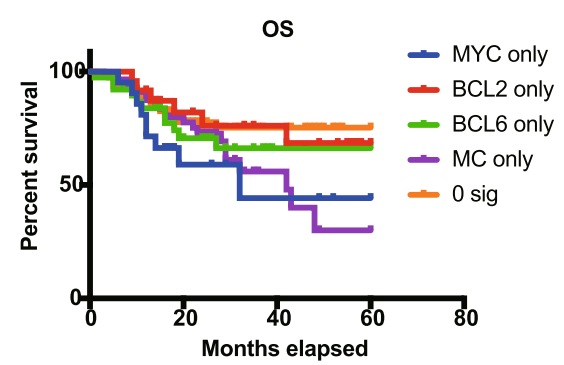

$\mathbf{F}$

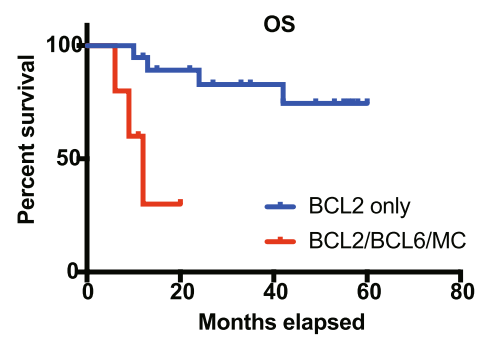

Fig. 4 survival analyses of de novo cases that received R-CHOP or CHOP-like chemotherapy. a-b Kaplan-Meier plot for 5-year OS and PFS, respectively, according to the signature numbers carried by each case (3 signatures or 0 signature). c-d Kaplan-Meier plot for 5-year OS and PFS, respectively. Each case carried a single or no signature. Cases were grouped according to the signature type (MYC-trans, BCL2-trans, BCL6-trans or MC-trans). e-f Kaplan-Meier plot for 5-year OS and PFS, respectively. Patients grouped in EZB subtype were classified by signature type (BCL2-trans only or BCL2-trans plus BCL6-trans or MC-trans)

limited sample number. As has been described in previous reports, the majority of $C D 79 B$ variants including hotspot Y196 clustered in ITAM domain, which were related with NF- $\mathrm{kB}$ pathway activation. Our result was consistent with the above findings as the majority of cases bearing $C D 79 B$ mutations were classified into nonGCB subtype. Moreover, it was worth mentioning that in our study we reported for the first time that a series of intron splice-site were identified as recurrent variants in DLBCL patients (e.g. c.550-1G > A, c.550-1G > C, c.550-3_552del, etc.). Our results further indicated that such splice-site variants probably result in CD79B protein truncating, causing $C D 79 B$ dysfunction in a unique way compared with $C D 79 B^{\mathrm{Y} 196}$ variant. Furthermore, co-mutation analysis indicated significantly difference compared with classical $C D 79 B{ }^{\mathrm{Y} 196}$ variant in terms of variant co-occurrence with $M Y D 88^{\mathrm{L} 265 \mathrm{P}}$. COO classification also demonstrated differences in DLBCL patients carrying $C D 79 B$ splice-site variants, in comparison of those with $C D 79 B{ }^{\mathrm{Y} 196}$ variant.
In summary, our results provided evidences that such obviously different mutation pattern of $C D 79 B$ splicesite variants suggested differential impact on DLBCL pathogenesis. However, the exact impact of CD79B truncating protein on the physiological signal transduction of NF-kB pathway and DLBCL pathogenesis calls for further functional study.

Until now, comprehensive studies have revealed that the genetic landscape of DLBCL is heterogeneous, which aids in our understanding of oncogenic mechanisms and provides novel insight into exploring better treatment strategies. To date, Schmitz et al. and Chapuy et al. showed that most DLBCL cases could be subcategorized into several distinct subsets, each of which had unique clinical, molecular and transcriptional characteristics. However, there is still inevitable heterogeneity within each group in their models. Nevertheless, if all expanding factors were taken into consideration, the system would gradually become too complicated to apply in routine clinical scenario. Therefore, in this study, instead 
of mutually exclusive classification, we aimed to define several non-mutually exclusive genetic signatures to describe and understand the complex molecular features of DLBCL, based on molecular information that was feasible to obtain including $M Y C / B C L 2 / B C L 6$ translocations as well as mutation data from a limited panel of genes. In this study, based on previous research methods and results, we preliminarily determined four genetic signatures using a machine learning-based algorithm. In addition, through analysis of prognostic data based on their signature types, we demonstrated unique cumulative prognostic impact based on the number of signatures each patient carry. Therefore, this model is applicable for target-oriented in therapeutic decision making and prognostic evaluation.

Notably, in this study, some of cases with single genetic signature also carry mutations commonly identified in other B cell malignancies. For those carrying single $M Y C$-trans signatures, 8 of 20 cases were affected by ID3-TCF3-CCND3 pathway mutations, which were prevalent in Burkitt lymphoma (BL) [27-29]. For cases with single $B C L 2$-trans signature, a significant proportion $(21 / 23,91.3 \%)$ of cases harbor gene mutations in chromatin modification, including KMT2D, CREBBP, $E P 300$, and $E Z H 2$, and several other signaling pathways (STAT6, SOCS1, TNFRSF14) which were similar to the genetic feature described in follicular lymphoma [30]. In addition, for cases with single BCL6-trans signature, the mutations in several genes were also frequently determined in marginal zone lymphoma [31-33], including NOTCH2 (14.6\%, 6/41), KLF2 (34.1\%, 14/41), TNFAIP3 (19.5\%, 8/41), and FAS $(17.1 \%, 7 / 41)$ mutations.

It should be noted that, limited by gene panel and sample size, the genetic signatures of certain cases might be mislabeled by a RF prediction algorithm, and some other important genetic signatures might remain undiscovered in our study. Several other genetic alterations have already been revealed to be of potential importance in understanding the mechanism of pathogenesis, classification, therapeutic guidance, and prognosis evaluation but were not included in our set of genetic features (Supplementary Table S6). Additionally, due to the single-center nature of our high-throughput sequencing study, current study lack external data to further support our theories. However, we did aim to validate our model in an expanded-scale multi-centered study in future exploration. Considering the DLBCL genetic diversities among different human races, we believed that future research including multiple populations would provide more consolidated evidences. Our future work will focus on undertaking a multiplatform analysis of genetic features on expanding-scale cohort, so as to promoting the lymphoma signature landscape description and to facilitate the precise determination of genetic signature.

\section{Conclusion}

Unlike mutually exclusive molecular sub-classification, our observations supported novel insight into understanding complex genetic features by identifying the status of several non-mutually exclusive clustered genetic fingerprints. The identification of genetic signatures was also helpful for disease classification, but it was also expected to reveal actionable targets for novel therapy development and precise prognostic evaluation.

\section{Supplementary information}

Supplementary information accompanies this paper at https://doi.org/10. 1186/s12885-020-07198-1.

Additional file 1: Table S1. Patient characteristics and genetic variants information.

Additional file 2: Table S2. Gene list of our targeted NGS panel

Additional file 3: Table S7. Top 50 frequent variants included in the inhouse curated blacklist.

Additional file 4:. Supplementary Appendix

Additional file 5: Table S3. Variant list

Additional file 6: Figure S1. The mutation pattern in $283 \mathrm{DLBCL}$. The mutational heatmap indicated the genetic alterations in 239 de novo DLBCL, NOS, 25 relapsed DLBCL after R-CHOP or CHOP-like chemotherapy, and $19 \mathrm{tFL}$, ranked by their mutational frequencies in all cases. Color code by genetic alteration type: missense mutation or in-frame deletion/ insertion (blue), truncating mutation, splice donor/acceptor site mutation, or copy number loss in TP53 (red), SHM (yellow), translocation (orange), and nondetected (gray). COO classification was also indicated above the mutational heatmap. To the right of the mutational heatmap, the frequency of genetic alterations along with the negative $\log _{10} P$ value of the significance between de novo cases and relapsed/transformed cases are listed.

Additional file 7: Table S4. Binary matrix of the status of one-hot encoded genetic alterations, initial defined genetic signatures, and final convergent genetic signatures

Additional file 8: Table S5. Multivariate analysis including our set of four signatures and IPI score using enter method in cox proportional hazard regression model

Additional file $\mathbf{9}$ Table $\mathbf{S 6}$. Genetic alterations had been revealed to be of importance but not included in this study.

\section{Abbreviations}

DLBCL: Diffuse large B-cell lymphoma; CN: Copy Number; RF: Random Forest; ABC: Activated B-cell-like; NGS: Next-generation sequencing; SV: Structure variations; PCNSL: Primary central nervous system lymphoma; PMBL: Primary mediastinal large B-cell lymphoma; PCDLBCL-LT : Primary cutaneous DLBCL, leg type; HGBL, NOS : High-grade B-cell lymphoma, not otherwise specified; HGBL-DH/TH: HGBL with MYC and BCL2 and/or BCL6 translocations (); MHG: Molecular high-grade; tFL: Transformed follicular lymphoma; FFPE: Formalin-fixed paraffin embedded; IHC: Immunohistochemistry; FISH: Fluorescent in situ hybridization; OS: Overall survival „P PFS: Progressionfree survival; CDS: Coding sequences; AID: Activation-induced cytidine deaminase; SHM: Somatic hypermutation; CLL: Chronic lymphocytic leukemia

\section{Acknowledgments}

We thank all the faculty and staff in the Clinical and Laboratory Unit of the Department of Hematology, Tongji Hospital, Tongji Medical College, Huazhong University of Science and Technology for their clinical and technical support.

\section{Authors' contributions}

WZ designed the study, wrote the manuscript, performed bioinformatic analysis. LY revised the manuscript, designed and performed survival and 
comparison analysis in this study. YQG collected clinical sample and related data. KFS performed high-throughput sequencing experiment. MLZ performed high-throughput sequencing experiment. HDC performed highthroughput sequencing experiment. JCW collected clinical sample and related data. YW performed FISH experiment and gather related data. LW performed statistical analysis. YC performed statistical analysis. NW conducted clinical data analysis. XHT conducted clinical data analysis. KHY oversighted the whole study and provide professional consultation. MX designed the study and oversight the study progress. JFZ oversighted the study and provide professional consultation. All authors stated that they have read and approved the manuscript submission.

\section{Funding}

This work was funded by grants from the National Science Foundation of China (No.81770211, No. 81700145). Both grants provided fundamental support for the bioinformatic data analysis, statistical clinical data investigation and manuscript preparations.

\section{Availability of data and materials}

The clinical and genetic variants data analyzed in this study are provided in this article and in supplementary files. The raw datasets analyzed during the current study are available from the corresponding author on reasonable request.

\section{Ethics approval and consent to participate}

This study was approved by the Ethics Committee of Tongji Hospital. All patients have known the details of our project and signed an Informed Consent Form.

\section{Consent for publication}

Not applicable.

\section{Competing interests}

The authors in this article declare that they have no conflict of interest.

\section{Author details}

${ }^{1}$ Department of Hematology, Tongji Hospital, Tongji Medical College, Huazhong University of Science and Technology, Wuhan 430030, Hubei Province, P.R. China. 'Department of Hematology/Oncology, Guangxi Medical University Cancer Hospital, No. 71 Hedi Road, Nanning, Guangxi 530021, P.R. China. ${ }^{3}$ Department of Pathology, The University of Duke, Durham, North Carolina, USA.

\section{Received: 12 March 2020 Accepted: 20 July 2020}

Published online: 31 July 2020

\section{References}

1. Swerdlow SH, Campo E, Pileri SA, Harris NL, Stein H, Siebert R, et al. The 2016 revision of the World Health Organization classification of lymphoid neoplasms. Blood. 2016;127:2375-90.

2. Coiffier B. Rituximab therapy in malignant lymphoma. Oncogene. 2007;26: 3603-13.

3. Reddy A, Zhang J, Davis NS, Moffitt AB, Love CL, Waldrop A, et al. Genetic and Functional Drivers of Diffuse Large B Cell Lymphoma. Cell. 2017;171: 481-494.e15.

4. Schmitz R, Wright GW, Huang DW, Johnson CA, Phelan JD, Wang JQ, et al. Genetics and pathogenesis of diffuse large B-cell lymphoma. N Engl J Med. 2018;378:1396-407.

5. Chapuy B, Stewart C, Dunford AJ, Kim J, Kamburov A, Redd RA, et al. Molecular subtypes of diffuse large B cell lymphoma are associated with distinct pathogenic mechanisms and outcomes. Nat Med. 2018;24:679-90.

6. Alizadeh AA, Eisen MB, Davis RE, Ma C, Lossos IS, Rosenwald A, et al. Distinct types of diffuse large B-cell lymphoma identified by gene expression profiling. Nature. 2000;403:503-11.

7. Gonzalez-Aguilar A, Idbaih A, Boisselier B, Habbita N, Rossetto M, Laurenge $A$, et al. Recurrent mutations of MYD88 and TBL1XR1 in primary central nervous system lymphomas. Clin Cancer Res. 2012;18:5203-11.

8. Mottok A, Hung SS, Chavez EA, Woolcock B, Telenius A, Chong LC, et al. Integrative genomic analysis identifies key pathogenic mechanisms in primary mediastinal large B-cell lymphoma. Blood. 2019;134:802-13.
9. Koens L, Zoutman WH, Ngarmlertsirichai P, Przybylski GK, Grabarczyk P, Vermeer $\mathrm{MH}$, et al. Nuclear factor-kappaB pathway-activating gene aberrancies in primary cutaneous large B-cell lymphoma, leg type. J Investigative Dermatol. 2014;134:290-2.

10. Bouska A, Bi C, Lone W, Zhang W, Kedwaii A, Heavican T, et al. Adult highgrade B-cell lymphoma with Burkitt lymphoma signature: genomic features and potential therapeutic targets. Blood. 2017;130:1819-31.

11. Ennishi D, Jiang A, Boyle M, Collinge B, Grande BM, Ben-Neriah S, et al. Double-hit gene expression signature defines a distinct subgroup of germinal center B-cell-like diffuse large B-cell lymphoma. J Clin Oncol. 2019; 37:190-201.

12. Sha C, Barrans S, Cucco F, Bentley MA, Care MA, Cummin T, et al. Molecular high-grade B-cell lymphoma: defining a poor-risk group that requires different approaches to therapy. J Clin Oncol. 2019;37:202-12.

13. Hans CP, Weisenburger DD, Greiner TC, Gascoyne RD, Delabie J, Ott G, et al. Confirmation of the molecular classification of diffuse large B-cell lymphoma by immunohistochemistry using a tissue microarray. Blood. 2004; 103:275-82.

14. Rimsza LM, Wright G, Schwartz M, Chan WC, Jaffe ES, Gascoyne RD, et al. Accurate classification of diffuse large B-cell lymphoma into germinal center and activated B-cell subtypes using a nuclease protection assay on formalin-fixed, paraffin-embedded tissues. Clin Cancer Res. 2011;17:3727-32.

15. DAWSON IM, CORNES JS, MORSON BC. Primary malignant lymphoid tumours of the intestinal tract. Report of 37 cases with a study of factors influencing prognosis. Br J Surg. 1961;49:80-9.

16. Cheson BD, Fisher RI, Barrington SF, Cavalli F, Schwartz LH, Zucca E, et al. Recommendations for initial evaluation, staging, and response assessment of Hodgkin and non-Hodgkin lymphoma: the Lugano classification. J Clin Oncol. 2014;32:3059-68.

17. Gebauer N, Bernard V, Feller AC, Merz H. ID3 mutations are recurrent events in double-hit B-cell lymphomas. Anticancer Res. 2013;33:4771-8.

18. Panea Rl, Love CL, Shingleton JR, Reddy A, Bailey JA, Moormann AM, et al. The whole-genome landscape of Burkitt lymphoma subtypes. Blood. 2019; 134:1598-607.

19. Okosun J, Wolfson RL, Wang J, Araf S, Wilkins L, Castellano BM, et al. Recurrent mTORC1-activating RRAGC mutations in follicular lymphoma. Nat Genet. 2016;48:183-8.

20. Clipson A, Wang M, de Leval L, Ashton-Key M, Wotherspoon A, Vassiliou G, et al. KLF2 mutation is the most frequent somatic change in splenic marginal zone lymphoma and identifies a subset with distinct genotype. Leukemia. 2015;29:1177-85.

21. Wang K, Li M, Hakonarson H. ANNOVAR: functional annotation of genetic variants from high-throughput sequencing data. Nucleic Acids Res. 2010;38:e164.

22. Khodabakhshi AH, Morin RD, Fejes AP, Mungall AJ, Mungall KL, BolgerMunro $M$, et al. Recurrent targets of aberrant somatic hypermutation in lymphoma. Oncotarget. 2012;3:1308-19.

23. de Miranda NFCC, Georgiou K, Chen L, Wu C, Gao Z, Zaravinos A, et al. Exome sequencing reveals novel mutation targets in diffuse large B-cell lymphomas derived from Chinese patients. Blood. 2014;124:2544-53.

24. Karube K, Enjuanes A, Dlouhy I, Jares P, Martin-Garcia D, Nadeu F, et al. Integrating genomic alterations in diffuse large B-cell lymphoma identifies new relevant pathways and potential therapeutic targets. Leukemia. 2018; 32:675-84.

25. Puente XS, Pinyol M, Quesada V, Conde L, Ordonez GR, Villamor N, et al. Whole-genome sequencing identifies recurrent mutations in chronic lymphocytic leukaemia. Nature. 2011;475:101-5.

26. Jardin F, Pujals A, Pelletier L, Bohers E, Camus V, Mareschal S, et al. Recurrent mutations of the exportin 1 gene (XPO1) and their impact on selective inhibitor of nuclear export compounds sensitivity in primary mediastinal Bcell lymphoma. Am J Hematol. 2016;91:923-30.

27. Schmitz R, Young RM, Ceribelli M, Jhavar S, Xiao W, Zhang M, et al. Burkitt lymphoma pathogenesis and therapeutic targets from structural and functional genomics. Nature. 2012;490:116-20.

28. Love C, Sun Z, Jima D, Li G, Zhang J, Miles R, et al. The genetic landscape of mutations in Burkitt lymphoma. Nat Genet. 2012;44:1321-5.

29. Lopez C, Kleinheinz K, Aukema SM, Rohde M, Bernhart SH, Hubschmann D, et al. Genomic and transcriptomic changes complement each other in the pathogenesis of sporadic Burkitt lymphoma. Nat Commun. 2019;10:1459.

30. Krysiak K, Gomez F, White BS, Matlock M, Miller CA, Trani L, et al. Recurrent somatic mutations affecting $B$-cell receptor signaling pathway genes in follicular lymphoma. Blood. 2017;129:473-83. 
31. Rossi D, Trifonov V, Fangazio M, Bruscaggin A, Rasi S, Spina V, et al. The coding genome of splenic marginal zone lymphoma: activation of $\mathrm{NOTCH} 2$ and other pathways regulating marginal zone development. J Exp Med. 2012;209:1537-51.

32. Spina V, Khiabanian H, Messina M, Monti S, Cascione L, Bruscaggin A, et al. The genetics of nodal marginal zone lymphoma. Blood. 2016;128:1362-73.

33. Zhang Q, Siebert R, Yan M, Hinzmann B, Cui X, Xue L, et al. Inactivating mutations and overexpression of $B C L 10$, a caspase recruitment domaincontaining gene, in MALT lymphoma with t $(1 ; 14)$ (p22; 932$)$. Nat Genet. 1999;22:63-8.

\section{Publisher's Note}

Springer Nature remains neutral with regard to jurisdictional claims in published maps and institutional affiliations.

Ready to submit your research? Choose BMC and benefit from:

- fast, convenient online submission

- thorough peer review by experienced researchers in your field

- rapid publication on acceptance

- support for research data, including large and complex data types

- gold Open Access which fosters wider collaboration and increased citations

- maximum visibility for your research: over $100 \mathrm{M}$ website views per year

At $\mathrm{BMC}$, research is always in progress.

Learn more biomedcentral.com/submissions 